Pacific Journal of Mathematic 


\title{
ANALYTIC INTERPOLATION OF CERTAIN MULTIPLIER SPACES
}

\author{
JAMES D. STAFNeY
}

\begin{abstract}
Let $W_{p}$ denote the space of all functions on the circle which are the uniform limit of a sequence of trigonometric polynomials which is bounded as a sequence of multipliers for $l_{p}, 1 \leqq p \leqq 2$. Let $U_{s}$ be the interpolation space $\left[W_{2}, W_{1}\right]_{s}$ (see 1.1). Our main result, Theorem 2.4, states that for a compact subset $E$ of the circle, $U_{s} \mid E=C(E)$ if and only if $W_{1} \mid E=C(E)$. A major step in the proof is a maximum principle for interpolation, Theorem 1.7. We also give a direct proof that $U_{s} \neq W_{p}$ (see Theorem 2.7) for corresponding $s$ and $p$.
\end{abstract}

1. Some properties of analytic interpolation.

1.1. Let $B^{0}$ and $B^{1}$ be two Banach spaces continuously embedded in a topological vector space $V$ such that $B^{0} \cap B^{1}$ is dense in both $B^{0}$ and $B^{1}$. For $0<s<1$, let $\widetilde{F},\left[B^{0}, B^{1}\right]_{s}$ and $B^{0}+B^{1}$ denote the spaces as defined in $[1, \S 1]$. For two Banach spaces $X$ and $Y$ we let $O(X, Y)$ denote the Banach space of bounded linear operators from $X$ into $Y$ where the norm is the usual operator norm. Let $O(X)$ denote $O(X, X)$.

1.2. Assume the notation and conditions of paragraph 1.1 and for convenience let $B_{s}$ denote the space $\left[B^{0}, B^{1}\right]_{s}, 0<s<1$. Let $V^{\prime}$ denote the Banach space

$$
O\left(B^{\circ} \cap B^{1}, B^{0}+B^{1}\right) .
$$

Let $A_{j}$ be a closed subspace of $O\left(B^{j}\right), j=0,1$. By restricting the elements in $A_{j}$ to $B^{0} \cap B^{1}$ in the obvious way we may regard $A_{j}$ as continuously embedded in the topological vector space $V^{\prime}$, and it is with respect to this embedding that we understand $\left[A_{0}, A_{1}\right]_{s}$; in particular, $\left[A_{0}, A_{1}\right]_{s}$ is a subspace of $V^{\prime}$. We will assume that $A_{0} \cap A_{1}$ is dense in $A_{j}$ with respect to the norm of $A_{j}, j=0,1$, when these spaces are embedded in $V^{\prime}$ as described. Since $B^{\circ} \cap B^{1}$ is dense in $B^{0}$ and $B^{1}$, we know from $[1, \S 9.3]$ that $B^{0} \cap B^{1}$ is dense in $B_{s}$; thus, since $B_{s} \subset B^{0}+B^{1}$, the restriction of elements of $O\left(B_{s}\right)$ to $B^{0} \cap B^{1}$ gives a continuous embedding of $O\left(B_{s}\right)$ in $V^{\prime}$ in the obvious manner. Note that each element of $A_{0} \cap A_{1}$ is bounded with respect to the norm \|\|$_{B_{s}}$ restricted to $B^{0} \cap B^{1}$ and is, therefore, contained in the enbedded $O\left(B_{s}\right)$. Let $A_{s}$ denote the closure of $A_{0} \cap A_{1}$ in $O\left(B_{s}\right)$ where $O\left(B_{s}\right)$ is regarded as embedded in $V^{\prime}$ in the manner just described. Finally, we let $M_{s}$ and $N_{s}$ denote the norms of the spaces $A_{s}$ and $\left[A_{0}, A_{1}\right]_{s}$, respectively. 
Lemma 1.3. Assuming 1.2, $\left[A_{0}, A_{1}\right]_{s} \subset A_{s}$ and $M_{s} \leqq N_{s}, 0<s<1$.

This lemma is an immediate consequence of [1, $\S 11.1]$.

1.4. Assume the notation and conditions of 1.1. Let $J$ be a closed subspace of $B^{0}+B^{1}$. We will assume that

(1.4.1) $\quad I^{j}=J \cap B_{j}$, is closed in $B^{j}, j=0,1$. Clearly the map $\alpha$ defined by

$$
\alpha\left(x+I^{j}\right)=x+J \quad j=0,1
$$

is a continuous one to one linear map from $B^{j} / I^{j}$ into $V / J$. Let

$$
D_{s}=\left[\alpha\left(B^{0} / I^{\nu}\right), \alpha\left(B^{1} / I^{1}\right)\right]_{s} .
$$

Lemma 1.5. Assuming 1.4, if $x \in B_{s}, 0<s<1$, then $x+J \in D_{s}$ and

$$
\|x+J\|_{D_{s}} \leqq\left\|x+\left(J \cap B_{s}\right)\right\|_{B_{s}} /\left(J \cap B_{s}\right) .
$$

Proof. Let $x \in B_{s}, h \in J \cap B_{s}$ and $\varepsilon>0$. Choose $f \in \mathfrak{F}=\mathfrak{F}\left(B^{0}, B^{1}\right)$ such that $f(s)=x+h$ and

$$
\|f\|_{\mathfrak{Z}} \leqq \varepsilon+\|x+h\|_{B_{s}} .
$$

Let $g(\xi)=f(\xi)+J$ for $1 \leqq|\xi| \leqq \varepsilon$. Then it is clear that $g \in \mathfrak{F}_{1}$ where

$$
\widetilde{\mho}_{1}=\mathfrak{F}\left(\alpha\left(B^{0} / I^{0}\right), \alpha\left(B^{1} / I^{1}\right)\right)
$$

and that

$$
g(s)=x+J .
$$

Hence, $x+J \in D_{s}$. Furthermore, since it is clear that

$$
\|g\|_{\mathfrak{\mho}^{1}} \leqq\|f\|_{\mathfrak{F}},
$$

(1.5.1) follows from (1.5.2), (1.5.3), (1.5.4) and the fact that $h$ and $\varepsilon$ were chosen arbitrarily.

The following lemma can be proved by the usual method of successive approximations.

Lemma 1.6. Suppose that $D_{1}$ is a Banach space that is continuously embedded in a Banach space $D_{0}$ such that $D_{1}$ is dense in $D_{0}$ with respect to the norm of $D_{0}$. Suppose that there exist constants $c, c_{1}, c<1$, with the property that for each $x \in D_{1}$ there is a corresponding element $z$ in $D_{1}$ such that 


$$
|z|_{1} \leqq c_{1}|x|_{0} \quad \text { and } \quad|x-z|_{0} \leqq c|x|_{0} .
$$

Then $D_{1}=D_{0}$.

We will now establish a "maximum principle" for analytic interpolation.

THEOREM 1.7. If, in addition to the assumptions of paragraph $1.1, B^{0}=\left[B^{0}, B^{1}\right]_{s}$ for some $s(0<s<1)$, then $B^{0}=B^{1}$.

Proof. From the fact that $B^{0}$ and $B^{1}$ are continuously embedded in $V$ and the closed graph theorem we conclude that the norms $1 / 0$ and ||$_{s}$ on $B^{0}$ and $\left[B^{0}, B^{1}\right]_{s}$, respectively, are equivalent. In particular, there is a constant $c$ such that

$$
|x|_{0} \leqq c|x|_{s} \quad \text { for all } x \text { in } B^{0} .
$$

From $[1,9.4$. (ii)] we conclude that

$$
|x|_{s} \leqq|x|_{0}^{1-s}|x|_{1}^{s} \quad \text { for all } x \text { in } B^{0} \cap B^{1} .
$$

We conclude from (1.7.1) and (1.7.2) that

$$
|x|_{0} \leqq c^{1 / s}|x|_{1} \quad \text { for all } x \text { in } B^{0} \cap B^{1} .
$$

Thus, $B_{1}$ is continuously embedded in $B^{3}$. We shall now prove that (1.7.3) there is a constant $c_{1}$ with the property that for each $x$ in $B^{1}$ there is a corresponding $y$ in $B^{1}$ such that

$$
|y|_{1} \leqq c_{1}|x|_{0} \text { and }|y-x|_{0} \leqq(1 / 2)|x|_{0} .
$$

Let $x \in B^{1}$. In particular, $x \in\left[B^{0}, B^{1}\right]_{s}$ and, therefore, there exists an $f \in \mathfrak{F}\left(B^{0}, B^{1}\right)$ such that $f(s)=x$ and $|f|_{\mathfrak{F}\left(B^{0}, B^{1}\right)} \leqq 2|x|_{s}$. Since the norms ||$_{0}$ and ||$_{s}$ are equivalent we can choose a real number $\lambda$ so that $2|u|_{s} e^{\lambda s} \leqq(1 / 2)|u|_{0}$ for every $u$ in $B^{0}$. Let $g(\xi)=f(\xi) e^{-\lambda(\xi-s)}$ where $0 \leqq \operatorname{Re} \xi \leqq 1$. Then

$$
\begin{aligned}
x=g(s)= & \int_{-\infty}^{\infty} g(i t) \ell_{0}(s, t) d t \\
& +\int_{-\infty}^{\infty} g(1+i t) \mu_{1}(s, t) d t
\end{aligned}
$$

where $\mu_{0}$ and $\mu_{1}$ are the Poisson kernels for the strip $0 \leqq \operatorname{Re} \xi \leqq 1$ (see $[1,9.4])$. Let $y$ and $z$ denote the first and second integrals, respectively, appearing in (1.7.4). Since $\int_{-\infty}^{\infty}\left|\mu_{i}(s, t)\right| d t \leqq 1(i=0,1)$, $|g(i t)|_{0} \leqq 2|x|_{s} e^{\lambda s} \leqq(1 / 2)|x|_{0}$ (all real $\left.t\right)$, and

$$
|g(1+i t)|_{1} \leqq 2|x|_{s} e^{-\lambda(1-s)} \leqq(1 / 2) e^{-\lambda}|x|_{0}
$$


(all real $t$ ), it follows that $|x-z|_{0} \leqq(1 / 2)|x|_{0}$ and $|z|_{1} \leqq(1 / 2) e^{-2}|x|_{0}$. This proves (1.7.3). Since $B^{1}$ is continuously embedded as a dense subspace in $B^{n}$ and (1.7.3) holds, the conclusion of Theorem 1.7 follows from Lemma 1.6.

2. The spaces $W_{p}$ and $U_{s}$. Let $l_{p}, 1 \leqq p<\infty$, denote the Banach space of complex valued functions $x$ on the integers such that

$$
\|x\|_{l_{p}}=\left(\sum|x(n)|^{p}\right)^{1 / p}<\infty
$$

where the sum is over all integers $n$. Each function $\alpha$ on the integers which vanishes outside some finite set determines a linear transformation $T_{a}$ on $l_{p}$, defined by

$$
T_{c} x(n)=\sum_{-\infty<k<\infty} x(n-k) \alpha(k) .
$$

Let $W_{p}^{\prime}$ denote the closure of the operators $T_{\alpha}$ in $O\left(l_{p}\right)$. Since $l_{1}$ is a dense subspace of each space $l_{p}, 1 \leqq p<\infty$, the restriction of elements in $O\left(l_{p}\right), 1 \leqq p \leqq 2$, to the subspace $l_{1}$ gives a one-to-one continuous linear embedding of $O\left(l_{p}\right), 1 \leqq p \leqq 2$, into the space

$$
R=O\left(l_{1}, l_{2}\right) \text {. }
$$

Throughout this section we will identify $O\left(l_{p}\right)$ with its image under this embedding without further comment. Let $U_{s}^{\prime}$ denote the space $\left[W_{2}^{\prime}, W_{1}^{\prime}\right]_{s}$ where $V$ in 1.1 is, in this case, $R$.

Our immediate purpose is to define a "Fourier transform" on $W_{p}^{\prime}$ and to prove Lemmas 2.2 and 2.3.

If $x$ is a complex valued function on the integers $Z$, let $\tau_{n} x(k)=$ $x(k-n)$. Let $\delta_{n}$ denote the function on $Z$ such that $\delta_{n}(n)=1$ and $\delta_{n}(k)=0, k \neq n$. If $x$ and $y$ are two complex valued function on $Z$ let

$$
x * y(m)=\sum_{n \in Z} x(m-n) y(n)
$$

define the function $x * y$ provided the sum converges absolutely for each $m \in Z$. For each $H$ in $W_{p}^{\prime}$ let $H^{\sim}$ denote the function $H\left(\delta_{0}\right)$ in $l_{p}$. The following lemma states the needed properties of the map $H \rightarrow H^{\sim}$. Note that $\tau_{n} x=\delta_{n} * x$ for each $n \in Z$ and for each complex valued function $x$ on $z$.

LeMma 2.1.

(2.1.1) $H \rightarrow H^{\sim}$ is a one-to-one linear transformation from $W_{p}^{\prime}$ into $l_{p}$.

(2.1.2) $H: x=H^{\sim} * x, H \in W_{p}^{\prime}, x \in l_{p}$.

(2.1.3) $(H K)^{\sim}=H^{\sim} * K^{\sim}, H, K \in W_{p}^{\prime}$.

Proof. The map $H \rightarrow H^{\sim}$ is clearly linear. Evidently, each $H$ in 
$W_{p}^{\prime}$ commutes with all operators $\tau_{m}, m \in Z$, since the operators of the form $T_{\alpha}$ commute with the operators $\tau_{m}, m \in Z$. Thus for $H \in W_{p}^{\prime}$ and $m \in Z$, we see that

$$
H\left(\delta_{m}\right)=H\left(\tau_{m} \delta_{0}\right)=\tau_{m} H\left(\delta_{0}\right)=\tau_{m} H^{\sim}=H^{\sim} * \delta_{m} .
$$

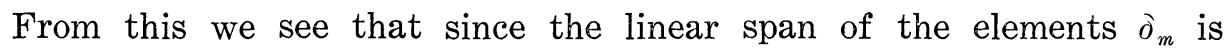
dense in $l_{p}$, the map $H \rightarrow H^{\sim}$ is one-to-one. Obviously, $H^{\sim}$ is in $l_{p}$. To establish (2.1.2) we first note that since $H^{\sim}$ is in $l_{q}\left(q^{-1}+p^{-1}=1\right)$ the map $x \rightarrow H^{\sim} * x$ is a continuous linear map from $l_{p}$ into $c_{0}$, the space of complex valued functions on $Z$ which tend to 0 at $\pm \infty$. The map $x \rightarrow H x$ is also a continuous linear map from $l_{p}$ into $c_{0}$. These observations together with (2.1.4) and the density property of the $\delta_{m}$ 's noted above complete the proof of (2.1.2). To prove (2.1.3) we note that for $H$ and $K$ in $W_{p}^{\prime}, K^{\sim} \in l_{p}$, so by (2.1.2) we have

$$
H^{\sim} * K^{\sim}=H\left(K^{\sim}\right)=H\left(K \delta_{0}\right)=(H K) \delta_{0}=(H K)^{\sim} .
$$

This completes the proof of the lemma.

Let $L_{p}(1 \leqq p<\infty)$ denote the Banach space of measurable functions $g(\theta)$ on the circle (reals $\bmod 2 \pi)$ whose norm $\|g\|_{L_{p}}$,

$$
\|g\|_{L_{p}}=\left((1 / 2 \pi) \int_{0}^{2 \pi}|g(\theta)|^{1 / p} d \theta \mid\right)^{1 / p},
$$

is finite. Let $L_{\infty}$ denote the space of essentially bounded measurable functions $g$ with $\|g\|_{L_{\infty}}$ denoting the essential supremum of $g$.

Since each function $H^{\sim}, H \in W_{p}^{\prime}$, is in $l_{p}$, which is contained in $l_{2}$, there is a unique function $H^{\wedge}$ in $L_{2}$ such that $\sum H^{\sim}(n) e^{i n \theta}$ is the Fourier series of $H^{\wedge}$.

Lemma 2.2. For $1 \leqq p \leqq 2$ the map $H \rightarrow H^{\wedge}$ is a norm decreasing algebraic isomorphism from $W_{p}^{\prime}$ into $L_{\infty}$.

Proof. The fact that $H \rightarrow H^{\wedge}$ is a one-to-one linear map from $W_{p}^{\prime}$ into $L_{2}$ is clear from (2.1.1) and the fact that each function in $L_{2}$ is uniquely determined by its Fourier coefficients. For each $f \in L_{1}$, let $\lambda(f)$ denote the function on $Z$ defined by:

$$
\lambda(f)(n)=(1 / 2 \pi) \int_{0}^{2 \pi} f(\theta) e^{-i n \rho} d \theta .
$$

It is clear from the Schwarz inequality that the map $(f, g) \rightarrow \lambda(f \cdot g)(n)$ is a continuous bilinear functional on $L_{2} \oplus L_{2}$ for each integer $n$. On the other hand, the map

$$
(f, g) \rightarrow(\lambda(f) * \lambda(g))(n)
$$


is also a continuous bilinear functional on $L_{2} \oplus L_{2}$. Since these functionals (for each $n$ ) clearly agree when $f$ and $g$ are trigonometric polynomials, they must agree on $L_{2} \oplus L_{2}$. Since $\lambda$ is a one-to-one map, the multiplicative property of $H \rightarrow H^{\wedge}$ now follows from (2.1.3). To prove that the map is norm decreasing we first note the following inequalities:

$$
\left\|H^{n}\right\|_{W_{p}^{\prime}} \geqq\left\|H^{n} \delta_{0}\right\|_{l_{p}}=\left\|\left(H^{n}\right)^{\sim}\right\|_{l_{p}} \geqq\left\|\left(H^{n}\right)^{\sim}\right\|_{l_{2}}=\left\|\left(H^{n}\right)^{\wedge} .\right\|_{L_{2}}=\left\|\left(H^{\wedge}\right)^{n}\right\|_{L_{2}} .
$$

It is well known that $\left(\left\|H^{n}\right\|_{W_{p}^{\prime}}\right)^{1 / n}$ converges to the spectral radius of $H$, which is dominated by $\|H\|_{W_{p}^{\prime}}$, and that $\left(\left\|\left(H^{\wedge}\right)^{n}\right\|_{L_{2}}\right)^{1 / n}$ converges to $\left\|H^{\wedge}\right\|_{L_{\infty}}$ as $n \rightarrow \infty$. This proves the lemma.

Let $W_{p}$ and $U_{s}$ denote the functions on the circle of the form $H^{\wedge}$ where $H \in W_{p}^{\prime}, U_{s}^{\prime}$, respectively. The following lemma is an immediate consequence of Lemma 2.2 .

LEMMA 2.3. $W_{p}$ consists precisely of the functions on the circle which are the uniform limits of sequences $H_{n}^{\wedge}$ of trigonometric polynomials such that $H_{n}$ is a Cuachy sequence in $W_{p}^{\prime}$.

For any subset $E$ of the circle group $U_{s} \mid E$ denotes the functions on $E$ obtained by restricting the functions of $U_{s}$ to $E$ and $C(E)$ denotes the continuous complex valued functions on $E$.

THEOREM 2.4. Suppose that $E$ is a compact subset of the circle group and $0<s<1$. Then $U_{s} \mid E=C(E)$ if and only if $W_{1} \mid E=C(E)$.

Proof. First assume that $W_{1} \mid E=C(E)$. By Lemma 1.3, $U_{s}^{\prime} \subset W_{p}^{\prime}$; consequently, $U_{s} \subset W_{p}$. We conclude from Lemma 2.3 that $W_{p} \subset C(T)$. Thus, $U_{s} \mid E \subset C(E)$. Since $W_{2}^{\prime} \supset W_{1}^{\prime}$, it is clear from the definition of interpolation that $U_{s}^{\prime} \supset W_{1}^{\prime}$. Thus, $U_{s} \mid E \supset C(E)$.

Consider the converse and assume that $U_{s} \mid E=C(E)$. In 1.4 we let $B^{0}=W_{2}^{\prime}, B^{1}=W_{1}^{\prime}, V=R$ and

$$
J=\left\{a \in W_{2}^{\prime}: \widehat{a}(\theta)=0, \theta \in E\right\} .
$$

The assumptions on $J$ in 1.4 are clearly satisfied since by Lemma 2.2, the maps $a \rightarrow \widehat{a}$ are continuous on $W_{1}^{\prime}$ and $W_{2}^{\prime}$. By Theorem 1.5, if $x \in U_{s}^{\prime}$, then $x+J$ is in the space

$$
\left[\alpha\left(W_{2}^{\prime} / J\right), \alpha\left(W_{1}^{\prime} /\left(J \cap W_{1}^{\prime}\right)\right)\right]_{s} .
$$

However, by hypothesis, the cosets in $V$ of the form $x+J,, x \in U_{s}^{\prime}$, are the same as the cosets $y+J, y \in W_{2}^{\prime}$. Therefore, the space in (2.4.1) is $\alpha\left(W_{2}^{\prime} / J\right)$. Since $W_{2}^{\prime} \supset W_{1}^{\prime}$,

$$
\alpha\left(W_{2}^{\prime} / J\right) \supset \alpha\left(W_{1}^{\prime} /\left(J \cap W_{1}^{\prime}\right)\right) ;
$$


therefore, we conclude from 1.7 that

$$
\alpha\left(W_{2}^{\prime} / J\right)=\alpha\left(W_{1}^{\prime} /\left(J \cap W_{1}^{\prime}\right)\right) ;
$$

or, what is the same thing, that $W_{1} \mid E=C(E)$. This completes the proof.

Comment 2.5. It is natural to compare $U_{s}$ and $W_{p}$ where $\left[l_{2}, l_{1}\right]_{s}=$ $l_{p}$, i.e., $(1-s) / 2+s=1 / p$. In [3] we showed that Theorem 2.4 is not valid for $W_{p}$. To be exact, there is a compact subset $E$ of the circle such that $W_{p}\left|E \neq C(E)=W_{4 / 3}\right| E, 1 \leqq p<4 / 3$. We had originally used this result to show that $W_{p} \neq U_{s}$; however, the referee has suggested a direct proof which we will now give.

Lemma 2.6. Let $h_{n}$ be a sequence in $U_{s}, 0<s<1$, such that $\left\|h_{n}\right\|_{s} \leqq M$ (here \|\|$_{s}$ is the norm in $U_{s}$ ) and $h_{n} \rightarrow h$ almost everywhere. Then $h$ agrees with some continuous function almost everywhere.

Proof. Since $\left\|h_{n}\right\|_{s} \leqq M$ there exist functions $f_{n}(\theta, \xi)$, analytic in $\xi$ for $0<B(\xi)<1$ and continuouns in $0 \leqq B(\xi) \leqq 1$, such that for any real number $t,\left\|f_{n}(\theta, i t)\right\|_{0} \leqq 2 M,\left\|f_{n}(\theta, 1+i t)\right\|_{1} \leqq 2 M$ and $f_{n}(\theta, s)=$ $h_{n}(\theta)$. Let $g_{n}(\theta, \xi)=f_{n}(\theta, \xi) e^{+\lambda(\xi-s)}$. Then

$$
\begin{aligned}
h_{n}(\theta)=f_{n}(\theta, s)=g_{n}(\theta, s)= & \int_{-\infty}^{+\infty} g_{n}(\theta, i t) \mu_{0}(s, t) d t \\
& +\int_{-\infty}^{+\infty} g_{n}(\theta, 1+i t) \mu_{1}(s, t) d t \\
= & u_{n}(\theta)+v_{n}(\theta)
\end{aligned}
$$

where $\mu_{0}$ and $\mu_{1}$ are the Poisson Kernels for the strip (see [1, 9.4]). Evidently $\left\|u_{n}\right\|_{0} \leqq 2 e^{-\lambda s} M,\left\|v_{n}\right\|_{1} \leqq 2 e^{\lambda(1-s)} M$. Since the $v_{n}$ are uniformly bounded, by taking a subsequence if necessary, we may assume that $v_{n}$ converges weakly to a bounded function $v(\theta)$, that is

$$
\lim _{n \rightarrow \infty} \int v_{n}(\theta) \varphi(\theta) d \theta=\int v(\theta) \varphi(\theta) d \theta
$$

for every integrable $\varphi$. Furthermore, as is readily seen, $v(\theta)$ belongs to $U_{1}$ and therefore is continuous. Since $h_{n}$ is uniformly bounded and converges almost everywhere, $h_{n}$ converges weakly. Since $h_{n}$ and $v_{n}$ converge weakly, $u_{n}$ converges weakly to some function $u$. From the fact that $\left|u_{n}(\theta)\right| \leqq\left\|u_{n}\right\|_{0} \leqq 2 e^{-\lambda s} M$, it follows that $|u(\theta)| \leqq 2 e^{-\lambda s}$ almost everywhere. Since $h=u+v$ almost everywhere and $\lambda$ can be taken arbitrarily large, $h$ agrees almost everywhere with the uniform limit of continuous functions. This completes the proof of the lemma. 
THEOREM 2.7. $U_{s}$ is properly contained in $W_{p}$ for $1<p<2$.

Proof. To prove the theorem it suffices to exhibit a sequence of functions in $U_{s}$ whose norms in $U_{s}$ tend to infinity and whose norms in $W_{p}$ remain bounded. Let $h\left(e^{i t}\right)=1$ for $0 \leqq t \leqq \pi$ and $h\left(e^{i t}\right)=0$ for $\pi<t<2 \pi$. Then $h$ is a multiplier for $l_{p}$ (see [2]), which does not agree almost everywhere with any continuous function. Let $\varphi_{n}$ be defined by: $\varphi_{n}\left(e^{i t}\right)=n$ for $|t| \leqq 1 / 2 n, \varphi_{n}\left(e^{2 t}\right)=0$ otherwise, $n=1,2, \cdots$. Let $h_{n}=h * \varphi_{n}, n=1,2, \cdots$. Since $\int_{0}^{2 \pi}\left|h_{n}\left(e^{i t}\right)\right| d t=1$, it follows that the $W_{p}$ norm of $h_{n}$ is the same as the $W_{p}$ norm of $h$; thus, $h_{n}$ is bounded in $W_{p}$. Since both $h$ and $\varphi_{n}$ belong to $L_{2}(0,2 \pi), h_{n} \in W_{1} \subset U_{s}$. Obviously, $h_{n}$ converges to $h$ almost everywhere. Since $h$ does not agree almost everywhere with any continuous function, it follows from Lemma 2.6 that $h_{n}$ is not bounded in $U_{s}$.

\section{BIBLIOGRAPHY}

1. A. P. Caldrón, Intermediate spaces and interpolation, the complex method, Studia Math. 24 (1964), 113-190.

2. I. I. Hirshmann, On multiplier transformations, Duke Math. J. 26 (1959), 221-242.

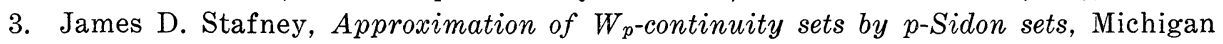
Math. J. 16 (1969), 161-176.

Received August 26, 1968.

University of California, Riverside 


\section{PACIFIC JOURNAL OF MATHEMATICS}

\section{EDITORS}

\author{
H. SAMELSON \\ Stanford University \\ Stanford, California 94305 \\ Richard Pierce \\ University of Washington \\ Seattle, Washington 98105
}

J. DUGUNDJI

Department of Mathematics

University of Southern California

Los Angeles, California 90007

BASIL GORDON*

University of California

Los Angeles, California 90024

\section{ASSOCIATE EDITORS}
E. F. BECKENBACH
B. H. NeUmanN
F. WOLF
K. YoSHIDA

\section{SUPPORTING INSTITUTIONS}

UNIVERSITY OF BRITISH COLUMBIA CALIFORNIA INSTITUTE OF TECHNOLOGY

UNIVERSITY OF CALIFORNIA MONTANA STATE UNIVERSITY

UNIVERSITY OF NEVADA

NEW MEXICO STATE UNIVERSITY

OREGON STATE UNIVERSITY

UNIVERSITY OF OREGON

OSAKA UNIVERSITY

UNIVERSITY OF SOUTHERN CALIFORNIA
STANFORD UNIVERSITY

UNIVERSITY OF TOKYO

UNIVERSITY OF UTAH

WASHINGTON STATE UNIVERSITY

UNIVERSITY OF WASHINGTON

AMERICAN MATHEMATICAL SOCIETY CHEVRON RESEARCH CORPORATION TRW SYSTEMS

NAVAL WEAPONS CENTER 


\section{Pacific Journal of Mathematics}

\section{Vol. 32, No. $1 \quad$ January, 1970}

Robert Alexander Adams, Compact Sobolev imbeddings for unbounded domains ........................................ 1

Bernhard Amberg, Groups with maximum conditions .................. 9

Tom M. (Mike) Apostol, Möbius functions of order k............... 21

Stefan Bergman, On an initial value problem in the theory of two-dimensional transonic flow patterns ................... 29

Geoffrey David Downs Creede, Concerning semi-stratifiable spaces ...... 47

Edmond Dale Dixon, Matric polynomials which are higher

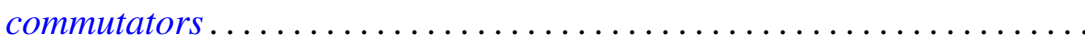

R. L. Duncan, Some continuity properties of the Schnirelmann density.

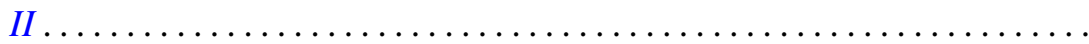

Peter Larkin Duren and Allen Lowell Shields, Coefficient multipliers of $H^{p}$

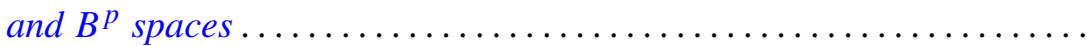

Hector O. Fattorini, On a class of differential equations for vector-valued

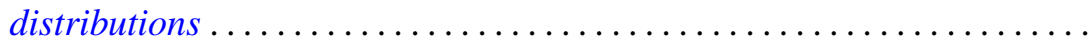

Charles Hallahan, Stability theorems for Lie algebras of derivations. . . . . . 105

Heinz Helfenstein, Local isometries of flat tori ................ 113

Gerald J. Janusz, Some remarks on Clifford's theorem and the Schur

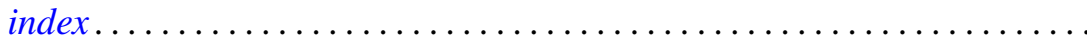

Joe W. Jenkins, Symmetry and nonsymmetry in the group algebras of discrete groups. ...

Herbert Frederick Kreimer, Jr., Outer Galois theory for separable

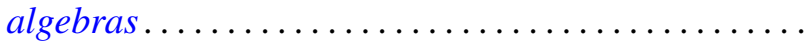

D. G. Larman and P. Mani, On visual hulls

R. Robert Laxton, On groups of linear recurrences. II. Elements of finite order.

Dong Hoon Lee, The adjoint group of Lie groups ...

James B. Lucke, Commutativity in locally compact rings

Charles Harris Scanlon, Rings of functions with certain Lipschitz

$$
\text { properties ............................... }
$$

Binyamin Schwarz, Totally positive differential systems .

James McLean Sloss, The bending of space curves into piecewise helical curves.

James D. Stafney, Analytic interpolation of certain multiplier spaces ...

Patrick Noble Stewart, Semi-simple radical classes.......

Hiroyuki Tachikawa, On left $\mathrm{QF}-3$ rings ...................... 255

Glenn Francis Webb, Product integral representation of time dependent nonlinear evolution equations in Banach spaces.... . . . 